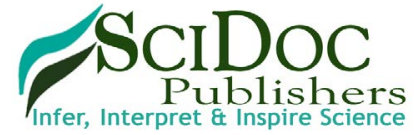

\title{
Does Cardiopulmonary Bypass Affect Lung Function In Orthotopic Lung Transplant Patients? (A 10 Year Institutional Retrospective Study) \\ Research Article
}

Jellish W. S*, Wilkinson N, Schwartz J M, Zaidi A

Department of Anesthesiology, Loyola University Medical Center, Maywood, IL, USA.

\section{Abstract}

Background: The use of Cardiopulmonary Bypass (CPB) in lung transplantation remains controversial. The goal of this study was to determine whether lung transplant with $\mathrm{CPB}$ affects lung function or development of bronchiolitis obliterans compared to off pump patients.

Methods: After IRB approval, a review of 151 patients who underwent orthotopic lung transplant with either CPB or off pump sequential placement was compared. Demographic data, bypass times, total surgery, intubation times and ICU stay were recorded. Biopsy determined rejection was compared, along with FEV1 changes from immediate post-transplant values. Significant differences between groups were determined at $\mathrm{p}<0.05$.

Results: Demographic variables were similar. CPB was associated with longer postoperative ventilation (4.8 vs 2.3 days) $\mathrm{p}=.07 .56 \%$ of bypass patients, vs $39 \%$ of non-bypass, showed no evidence of rejection. CPB patients also had less single rejection episodes $20 \%$ vs 30\%. FEV1 improved from baseline over the first year in both groups and improved over the next two years after transplant $(12.4,19.1,19.4 \mathrm{vs}, 7.3,11.2,10.6) \mathrm{CPB}$ vs non-bypass respectively $\mathrm{p}=0.034$.

Conclusion: Cardiopulmonary bypass appeared to have no deleterious effect on FEV1 or rejection. CPB may provide a protective mechanism with reduced rejection after lung transplantation.

Keywords : Lung Transplant; Cardiopulmonary Bypass; Lung Function; Bronchiolitis Obliterans; Gastroesophageal Reflux.

\section{Introduction}

Long-term survival following orthotopic lung transplantation is affected by the development ofchronic lung allograft dysfunction which would include bronchiolitis obliterans syndrome and restrictive allograft dysfunction. This affects $50-60 \%$ of patients 5 years out from surgery and $90-100 \%$ by year 9 [1]. The pathogenesis of CLAD is poorly understood, but several possible mechanisms for the development of this disease process suggest that different inflammatory insults such as ischemia-reperfusion, infection and/or rejection can lead to the development of CLAD [2]. Acute cellular rejection is the most consistently described risk factor for allograft lung dysfunction [3]. Both acute rejection and lymphocyte bronchiolitis are associated with the increased risk of this syndrome. Attenuating autoimmune T-cell mediated damage to the lung allograft should reduce bronchial epithelial injury and fibro proliferative events [4]. Novel therapies aimed at even more aggressive $\mathrm{T}$-cell depletion with the use of alimentation and
anti-CD52 antibody has generated interest both as an induction agent and therapeutic agent to stabilize and improve pulmonary function [5].

Autoimmunity could also produce CLAD . Tissue injury exposes normally sequestered self-antigens and their fragments which act as triggers for auto reactive T-cell proliferation and antibody production. Type 5 collagen (Coll V) resides beneath the basement membrane of the perivascular and peribronchiolarlung tissue. Upon exposure to Coll (V) fragments, auto reactive T-cells, specific to Type 5 collagen, proliferate and secrete cytokines consistent with a Th1 response [6]. In patients with elevated Type 5 collagen cell mediated immunity, a 5-10 fold increased risk of high grade bronchiolitis obliterans was noted [7]. The use of cardiopulmonary bypass during lung transplant has also been associated with the development of $\mathrm{BO}$ [8]. Previous studies have predominantly found that the use of $\mathrm{CPB}$ for lung transplant may produce an inflammatory response that is

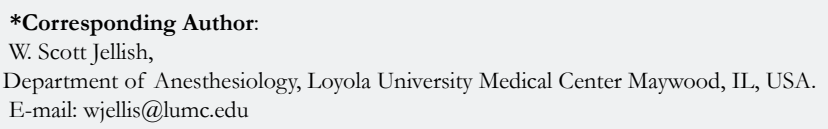

Copyright: Jellish W. $\mathbf{S}^{\circ}$ 2016. This is an open-access article distributed under the terms of the Creative Commons Attribution License, which permits unrestricted use, distribution and reproduction in any medium, provided the original author and source are credited. 
often indistinguishable from ischemia/reperfusion injury [8]. The integrity of the endothelium plays an integral role in this mechanism of lung injury by regulating the intervention between pulmonary vasodilation and vasoconstriction. This vasomotor dysfunction in the transplanted lung is associated with an acute rise in pulmonary vascular resistance [9]. During $\mathrm{CPB}$ the transplanted lung is reperfused with activated blood elements that may exacerbate this reperfusion injury [10]. Although it seems intuitive that $\mathrm{CPB}$, known to produce an inflammatory response and requires full systemic anticoagulation, would have a negative impact on function, there is still controversy over the extent to which CPB contributes to morbidity and death. Some have reported no adverse effects of $\mathrm{CPB}$ on length of intubation or duration of ICU stay [11]. In fact, some believe that the use of $\mathrm{CPB}$ reduces ischemic reperfusion injury during allograft implantation along with its immunosuppressive effects, both on a humoral level by depleting compliment factors and on a cellular level with a decrease in cell numbers of the innate and adaptive system, potentially improving survival. There have been few studies to date investigating the relationship between the use of $\mathrm{CPB}$ and the associated development of $\mathrm{BO}$.

This study seeks to determine if there is an association between $\mathrm{CPB}$ and the development of CLAD in orthotopic lung transplant patients.

\section{Materials and Methods}

After obtaining approval from the Institutional Review Board of Loyola University Chicago's Health Sciences Division (Protocol \#109643) on 8/27/07 to access patient records, 151 patients who underwent unilateral or bilateral orthotropic lung transplant from 2002-2012 were evaluated. The committee waived patient consent since the retrospective analysis did not submit any patient identifiers when reporting the findings of this study. The patients were chosen over this time frame because the surgical teams were similar and non-heparinized circuits were used until 2012 when Trillium Biosurface (Medtronic Inc. Minneapolis, MN55432) polymer coating with heparin was used on circuits and oxygenators. Patients were identified using the Department of Pulmonology and UNOS database. The patients were divided into two groups according to the use of cardiopulmonary bypass or if patients had sequential placement of the lungs without the use of bypass. Demographic information was collected from the patients, and the disease process which required lung transplantation was also recorded. Other information collected was cardiopulmonary bypass times, total surgery and anesthesia times, number of post-op days ventilated, and total time in the ICU. Patients were followed for three years and the number of patients who had a biopsy proven acute rejection was recorded. Transbronchial biopsies were performed at 1, 3, 6, 9 and 12 months post-transplant and upon clinical indication. This was continued at 15, 18 and 24 months if significant cellular rejection was found during the first year. Also, if patients had more than one episode of rejection as documented by symptoms after a 3 month symptom free period, the total number of rejections was recorded. Bronchiolitis obliterans syndrome is defined by the ISHLT as a greater than $20 \%$ decline in forced expiratory volume in 1 second or in forced expiratory flow, mid expiratory phase, in the absence of acute rejection or infection [12]. FEV-1 values were used as a surrogate to follow the development of bronchiolitis obliterans. Average percent change in FEV-1 was recorded in each year and overall change in FEV-1 was recorded in each patient over the three year study period. The patients were divided into two groups according to the use or absence of cardiopulmonary bypass. Demographic data were compared between the bypass or non-bypass group. Total surgery and anesthesia times were also compared between groups, as was total postoperative intubation and ICU times. Percentage of patients who had no biopsy evidence of rejection was compared between groups, as was the number who had one or more episodes of rejection. These were also compared between bypass and non-bypass groups. FEV-1 volume and percent change from immediate post bypass values were compared between groups. Patients who survived less than six months due to complications or rejection were excluded from the data set.

No power analysis was performed since this study evaluated all patients who underwent a single or double lung transplant over a 10 year period. Differences between bypass and non-bypass groups in numeric value were performed by t-test with Levene's test used for equality. Non-numeric values were compared using Fisher's Exact t-test.All values are expressed as mean $\pm \mathrm{SD}$ and $p$ $<0.05$ was used as the level to denote significance.

\section{Results}

One hundred and fifty one charts were reviewed with one hundred and forty seven patients being evaluated. Demographics and disease type for all patients are demonstrated in Table 1. The number of postoperative days ventilated was higher in patients who under went cardiopulmonary bypass (Table 2). Total time in the CV ICU tended to be longer in CPB patients but significant difference between the groups was not noted. The number of episodes of biopsy proven acute rejection was lower in the group that under went cardiopulmonary bypass compared to those that had sequential placement of the lungs without bypass (Table 3). A majority of patients with lung rejection were noted to have Grade A2 lung rejection patterns with $5 \%$ of patients demonstrating Grade B1R airway inflammation. $60 \%$ of the patients who underwent cardiopulmonary bypass had no evidence of acute rejection over a 3 year period compared to $30 \%$ of patients who did not receive CPB (Table 3). There also seemed to be more episodes of rejection over time in the group that did not undergo cardiopulmonary bypass (Table 3). FEV-1 was more improved from baseline at year one in the bypass group (Table 4). This stabilization in pulmonary function compared to baseline values was even more marked at year two after transplant in the bypass group compared to the non-bypass patients. By year three, FEV-1 plateaued in the bypass group and demonstrated a slight decrease in the non-bypass patients.

\section{Discussion}

This retrospective review of patients who have undergone orthotopic unilateral or bilateral lung transplant either with cardiopulmonary bypass or by sequential implantation without bypass demonstrates that in the early postoperative period patients who underwent their transplant using $\mathrm{CPB}$ actually experienced an improvement in lung function over baseline at year 1 and 2 which plateaued at year 3. They also had less episodes of acute rejection compared to non-bypass patients, 
Table 1. Demographic Data for Patients Undergoing Lung Transplant

\begin{tabular}{|c|c|c|}
\hline & Use of CPB & Absence of CPB \\
\hline $\mathrm{n}$ & 30 & 121 \\
\hline Average Age & $47.0 \pm 15.1$ & $51.6 \pm 12.5$ \\
\hline Total Males (\%) & 20 & 56 \\
\hline Total Females $(\%)$ & 10 & 65 \\
\hline Cystic Fibrosis & 5 & 13 \\
\hline Pulmonary Fibrosis + IPF & 17 & 40 \\
\hline COPD & 0 & 23 \\
\hline Emphysema & 1 & 26 \\
\hline $\mathrm{A} 1 \mathrm{~A}$ & 1 & 6 \\
\hline Sarcoidosis & 2 & 4 \\
\hline Scleroderma & 1 & 1 \\
\hline LAM & 1 & 3 \\
\hline Langerhans Histiocytosis & 0 & 1 \\
\hline Primary Graft Dysfunction & 0 & 0 \\
\hline BOS & 0 & 2 \\
\hline Bronchiectasis & 1 & 1 \\
\hline Other & 1 & 1 \\
\hline Single LTx & 2 & 77 \\
\hline Double & 8 & 44 \\
\hline
\end{tabular}

Table 2. Postoperative Days Ventilated and ICU Stay

\begin{tabular}{|c|c|c|}
\hline & Use of CPB & Absence of CPB \\
\hline Post-op Days Ventilated & $4.8 \pm 7.4$ & $2.3 \pm 4.3 \quad \mathrm{p}=0.002$ \\
\hline Days in ICU & $7.9 \pm 4.4$ & $4.1 \pm 2.9 \quad \mathrm{p}=0.16$ \\
\hline
\end{tabular}

Table 3. Incidence of Episodes of Acute Rejection - Percent of Subjects

\begin{tabular}{|c|c|c|c|c|c|c|}
\hline Use of CPB & 0 & 1 & 2 & 3 & 4 & 5 \\
\hline Yes & $60^{*}$ & 20 & 17 & 0 & 3 & 0 \\
\hline No & 38 & 29 & 15 & 11 & 5 & 2 \\
\hline
\end{tabular}

$*_{p}=0.023$

Table 4. FEV1 \% change from Baseline Values Year After Treatment

\begin{tabular}{|c|c|c|c|}
\hline & 1 & 2 & 3 \\
\hline Use of CPB & $12.5 \pm 3.2$ & $19.1 \pm 4.3^{*}$ & $19.4 \pm 5.9^{*}$ \\
\hline No CPB & $7.3 \pm 0.9$ & $11.2 \pm 1.5$ & $9.9 \pm 2.3$ \\
\hline
\end{tabular}

${ }^{*} p<0.05$ compared to No CPB

which suggests a possible immunosuppressive effect of cardiopulmonary bypass. These findings may also be attributable to a possible reduction in ischemic stress offered by continuous perfusion with cardiopulmonary bypass not observed with offpump transplant patients. Other investigators have demonstrated that cardiopulmonary bypass was associated with allograft dysfunction [13]. These investigators found that the patients who underwent $\mathrm{CPB}$ had poorer lung function with more pronounced infiltrate than the no CPB group. In addition, their intubation time was markedly longer,10 days, and the length of ICU stay was also prolonged. In our study, the bypass group was intubated longer than the off-pump group ( 5 days vs 2 days) respectively. But our intubation times were markedly reduced and more in line with other studies where CPB patients were intubated 5-6 days compared to 2 days in non-bypass transplant patients [1415]. This was probably related to diminished cardiopulmonary bypass times which averaged around 200 minutes in our study and diminished inflammatory response secondary to the disease state of the patient requiring lung transplantation. A large amount of the patients in the Gammie study had pulmonary hypertension, whereas other studies, including the present, had pulmonary fibrosis, cystic fibrosis or COPD as their primary diagnosis for 
transplant.

Several studies have demonstrated a positive effect of cardiopulmonary bypass on lung function afterlung transplantation [11]. With sequential lung transplantation, the first implanted allograft is subjected to increased ischemia-reperfusion injury during the implantation of the second allograft. This could occur from the entire cardiac output being diverted to the first implanted allograft, resulting in increased blood flow and pressure. The use of $\mathrm{CPB}$ ensures stable hemodynamics during implantation and facilitates difficult recipient dissection. One study found no significant difference in postoperative mechanical ventilation requirements between $\mathrm{CPB}$ and non-bypass groups [11]. Our study found a definite prolongation in mechanical ventilation in the CPB group, but the difference was only 2 days compared to other studies which showed similar differences in length of ventilation [14-15]. Survival after CPB was not different when compared to non-bypass patients after lung transplant. Triantafillou et al., [16] found no difference between $\mathrm{CPB}$ and non-bypass groups in time spent in the ICU, time to reach a $\mathrm{PO}_{2}$ of $>60 \mathrm{mmHg}$ on $\mathrm{RA}$ and survival. Survival was also similar between bypass the nonbypass lung transplant patients in another study by Hlozek [15]. In our study, it seemed that CPB improved overall outcomes by reducing the overall number of episodes of rejection compared to non-bypass patients and improving overall lung function with better FEV1 percent improvement over baseline at 1 and 2 years which was maintained in the third year. This finding seems to support the observation that $\mathrm{CPB}$ may be more beneficial than detrimental to overall outcomes after lung transplantation.

Study limitations are related to the fact that this is a retrospective analysis with a lack of control concerning anesthetic technique. Also, the use of transesophageal echocardiography and type of thoracotomy, which could affect the development of oropharyngeal dysphagia, were not controlled. Nevertheless, this study demonstrates that patients who underwent lung transplant with the use of cardiopulmonary bypass had minimal development of CLAD compared to those who had off-pump transplant. In addition, the clinical degree of rejection noted in all patients was mild and did not appreciably affect the FEV1 values noted during routine progress visits in these patients.

\section{Conclusions}

Our findings support the observation that cardiopulmonary bypass is a safe and effective method for lung transplantation and does not increase the risk of bronchiolitis obliterans or rejection in this patient population. Patients who underwent lung transplant with cardiopulmonary bypass actively experienced a transient increase in lung function during the second postoperative year with the episodes of rejection diminished. This suggests a possible immune suppressive effect with bypass or diminished ischemic stress to the lungs afforded by bypass as compared to off-pump sequential transplant.

\section{Acknowledgements}

MamdouhBakhos, Professor and Chair, Cardiothoracic Surgery, provided some of the data assessed in this report.

\section{References}

[1]. Christie JD, Edwards LB, Aurora P, Dobbels F, Kirk R, et al., (2008) Registry of the International Society for Heart and Lung Transplantation: Twenty-fifth Official Adult Lung and Heart/Lung Transplantation Report. J Heart Lung Transplant. 27(9): 957-969.

[2]. Hayes D, JR (2011) A review of bronchiolitis obliterans syndrome and therapeutic strategies. J Cardiothorac Surg. 6: 92-100.

[3]. Sharples LD, McNeil K, Stewart S, Wallwork J (2002) Risk factors for bronchiolitis obliterans: a systemic review of recent publications. J Heart Lung Transplant. 21(2): 271-281.

[4]. Todd JL, Palmer SM (2011) Bronchiolitis obliterans syndrome: The final frontier for lung transplantation. Chest. 149(2): 502-508.

[5]. Teuteberg JJ, Shullo MA, Zomak R, Toyoda Y, McNamara DM, et al., (2010) Alemtuzumab induction prior to cardiac transplantation with lower intensity maintenance immunosuppression: one year outcomes. Am J Transplant. 10(2): 382-388.

[6]. Sumpter TL, Wilkes DS (2004) Role of autoimmunity in organ allograft rejection: a focus on immunity to type $\mathrm{V}$ collagen in the pathogenesis of lung transplant rejection. Am J Physiol Lung Cell MolPhysiol. 286(6): L1129L1139.

[7]. Burlingham WJ, Love RB, Jankowska-Gan E, Haynes LD, Xu Q, et al., (2007) IL-17 dependent cellular immunity to collagen type $\mathrm{V}$ predisposes to obliterative bronchiolitis in human lung transplants. J Clin Invest. 117(11): 3498-3506.

[8]. Fullerton DA, Mitchell MB, McIntyre RC Jr, Banerjee A, Campbell DN, et al., (1993) Cold ischemia and reperfusion each produce pulmonary vasomotor dysfunction in the transplanted lung. J Thorac Cardiovasc Surg. 106: 1213-1217.

[9]. Downing TP, Sadeghi AM, Baumgartner WA, Reitz BA, Brackup A, et al., (1984) Acute physiologic changes following heart-lung allotransplantation in dogs. Ann Thorac Surg. 57: 479-483.

[10]. Donning SW, Edmonds LH (1992) Release of vasoactive substances during cardiopulmonary bypass. Ann Thorac Surg. 54: 1236-1243.

[11]. Szeto WY, Kreisel D, Karakousis GC, Pochettino A, Sterman DH, et al., (2002) Cardiopulmonary bypass for bilateral sequential lung transplantation in patients with chronic obstructive pulmonary disease without adverse effect on lung function or clinical outcome. J Thorac Cardiovasc Surg. 124: 241-249.

[12]. Estenne M, Maurer JR, Boehler A, Egan JJ, Frost A, et al., (2002) Bronchiolitis obliterans syndrome 2001: an update on the diagnostic criteria. J Heart Lung Transplant $21: 297-310$.

[13]. Gammie JS, Lee JC, Pham SM, Keenan RJ, Weyant RJ, et al., (1998) Cardiopulmonary bypass is associated with early allograft dysfunction but not death after double-lung transplant. J Thorac Cardiovasc Surg. 115: 990-997.

[14]. Moffatt-Bruce S, Karamichalis J, Robbins RC, White RI, Theodore J, et al., (2006) Are heart lung transplant recipients protected from developing bronchiolitis obliterans syndrome? Ann Thorac Surg. 81: 286-291.

[15]. Hlozek CC, Smedira NG, Kirby TJ, Patel AN, Pearl M (1997) Cardiopulmonary bypass (CPB) for lung transplantation. Perfusion. 12: 107-112.

[16]. Triantafillou AN, Pasque MK, Huddleston CB, Pond CG, Cerza RF, et al., (1994) Predictors, frequency and indications for cardiopulmonary bypass during lung transplantation in adults. Ann Thorac Surg. 57: 1248-1251. 